Genetic heterogeneity in depressive symptoms following the death of a spouse

\title{
Genetic heterogeneity in depressive symptoms following the death of a spouse: Polygenic score analysis of the US Health and Retirement Study
}

\author{
Benjamin W. Domingue $1 *$ \\ Hexuan Liu 2 \\ Aysu Okbay 3,4 \\ Daniel W. Belsky 5*
}

1. Stanford University Graduate School of Education

2. School of Criminal Justice at the University of Cincinnati

3. Department of Complex Trait Genetics, Vrije Universiteit, Center for Neurogenomics and Cognitive Research, Amsterdam, the Netherlands

4. Erasmus University Rotterdam Institute for Behavior and Biology, Rotterdam, the Netherlands

5. Duke University School of Medicine, Department of Medicine, Division of Geriatrics, Duke Univeristy Social Science Research Institute, Duke University Center for the Study of Aging and Human Development

* The corresponding authors can be contacted at bdomingue@stanford.edu and daniel.belsky@duke.edu.

Key words: Stressful life events, depression, polygenic score, genome-wide association study, diathesis-stress

Conflict of Interest: The authors report no conflict of interest. 
Genetic heterogeneity in depressive symptoms following the death of a spouse

\begin{abstract}
Experience of stressful life events is associated with risk of depression. Yet many exposed individuals do not become depressed. A controversial hypothesis is that genetic factors influence vulnerability to depression following stress. This hypothesis is most commonly tested with a "diathesis-stress" model, in which genes confer excess vulnerability. We tested an alternative model, in which genes may buffer against the depressogenic effects of life stress. We measured the hypothesized genetic buffer using a polygenic score derived from a published genome-wide association study (GWAS) of subjective wellbeing. We tested if married older adults who had higher polygenic scores were less vulnerable to depressive symptoms following the death of their spouse as compared to age-peers who had also lost their spouse and who had lower polygenic scores. We analyzed data from $\mathrm{N}=9,453$ non-Hispanic white adults in the Health and Retirement Study (HRS), a population-representative longitudinal study of older adults in the United States. HRS adults with higher wellbeing polygenic scores experienced fewer depressive symptoms during follow-up. Those who survived death of their spouses during follow-up $(n=1,829)$ experienced a sharp increase in depressive symptoms following the death and returned toward baseline over the following two years. Having a higher polygenic score buffered against increased depressive symptoms following a spouse's death. Effects were small and clinical relevance is uncertain, although polygenic score analyses may provide clues to behavioral pathways that can serve as therapeutic targets. Future studies of gene-environment interplay in depression may benefit from focus on genetics discovered for putative protective factors.
\end{abstract}


Genetic heterogeneity in depressive symptoms following the death of a spouse

\section{INTRODUCTION}

Experience of stressful life events is associated with risk of depression $(1,2)$. Yet many individuals exposed to stressful life events do not become depressed. A controversial hypothesis is that genetic differences between individuals modify the influence of stressful life events on risk of developing mental health conditions, including depression (3). This "diathesis-stress" hypothesis is supported by family-based genetic studies that find individuals with familial liability to depression are more vulnerable to developing depression following stress exposure $(4,5)$. Molecular genetic evidence for the diathesis-stress hypothesis from candidate-gene studies is contested, partly because of concerns about the candidate-gene approach (6). Genome-wide association studies (GWAS) offer opportunities to move beyond old arguments about candidate genes (7). GWAS discoveries for depression have yielded mixed results in tests of diathesisstress models (8-10). Here, we test proof of concept for using an alternative GWAS phenotype to develop a genetic measures of susceptibility to depression following stress. Instead of a diathesis, such as genetic liability to depression, we focused on a potential measure of psychological robustness to stress, the genetics of subjective wellbeing.

Subjective wellbeing represents a dimension of so-called "positive psychology," and may reflect the opposite end of an affective continuum from depression $(11,12)$. Like depression, subjective wellbeing is heritable $(13,14)$. A portion of this heritability is thought to reflect genetic influences shared with depression $(15,16)$. Theory predicts increased subjective wellbeing should buffer against deleterious consequences of stressful life events $(17,18)$.

We conducted a polygenic score study to test if genetic predisposition to greater subjective wellbeing buffered against risk of depression following experience of a stressful life 
Genetic heterogeneity in depressive symptoms following the death of a spouse

event, the death of one's spouse. We studied depressive symptoms in a cohort of older adults and their spouses followed longitudinally as part of the US Health and Retirement Study. We combined whole-genome single-nucleotide polymorphism (SNP) data already generated for the cohort and results from GWAS of subjective wellbeing in over 100,000 adults by the Social Science Genetic Association Consortium (16) to calculate cohort members's subjective wellbeing polygenic scores. Analysis tested if higher subjective-wellbeing polygenic score buffered survivors' risk of developing depressive symptoms following the death of their spouse.

\section{MATERIALS AND METHODS}

Sample. The Health and Retirement Study (HRS) is a longitudinal survey of a representative sample of Americans over the age of 50 and their spouses initiated in $1992(19,20)$. HRS is administered biennially and includes over 26,000 persons in 17,000 households. Respondents are interviewed about income and wealth, work and retirement, family and social connections, use of health services, and physical and mental health. A full description of the HRS is provided online at http://hrsonline.isr.umich.edu/index.php.

We linked HRS survey data from the "Rand Fat Files" (21) with genome-wide single nucleotide polymorphism (SNP) data downloaded from US National Institutes of Health Database of Genotypes and Phenotypes (dbGaP). HRS SNP genotyping was conducted by the NIH Center for Inherited Disease Research using DNA extracted from saliva collected during face-to-face interviews in HRS respondents' homes in 2006 and 2008 and Illumina HumanOmni 2.5 Quad BeadChip arrays. SNPs missing in $>5 \%$ of samples, with minor allele frequency $<1 \%$, or not in Hardy-Weinberg equilibrium $(\mathrm{p}<0.001)$ were removed. The final genetic database 
Genetic heterogeneity in depressive symptoms following the death of a spouse

included approximately 1.7M SNPs. HRS SNP data are described in detail at

http://hrsonline.isr.umich.edu/index.php?p=xxgen 1.

Our analysis is based on 9,453 HRS respondents (42\% male) who self-reported nonHispanic white race/ethnicity. Respondents were born between 1905-1975 and were 59.5 years old on average at first interview ( $\mathrm{IQR}=53-66)$. Included respondents participated in a median of 8 (IQR 6-10) follow-up assessments, yielding 74,512 total observations.

Analysis focused on non-Hispanic whites because this is the population in which the wellbeing GWAS was conducted and it is uncertain if a polygenic score derived from this GWAS will show comparable performance in populations with different ancestry (22-24). Because there are many fewer spousal pairs in the genetically-informed sample of non-white HRS respondents, we could not perform parallel analysis of the non-white sample.

\section{Measures}

Wellbeing Polygenic Score. We calculated HRS respondents' wellbeing polygenic scores based on the Social Science Genetic Association Consortium (SSGAC) GWAS of subjective wellbeing (16). The original GWAS included HRS respondents. To avoid upward bias to polygenic-scorewellbeing associations, SSGAC provided GWAS weights estimated from data excluding the HRS. Therefore, there was no overlap between the samples used to develop the polygenic score and the sample we analyzed. Polygenic scoring was conducted according to the methods described by Dudbridge (25) following the protocol used in previous work $(22,26,27)$. Briefly, we matched SNPs in the HRS genotype database with wellbeing GWAS SNPs. For each SNP, counts of wellbeing-associated alleles were weighted by GWAS-estimated effect-sizes.

Weighted counts were summed across SNPs to compute polygenic scores. 
Genetic heterogeneity in depressive symptoms following the death of a spouse

To account for any population stratification in our non-Hispanic white sample that might bias polygenic score-wellbeing associations, we computed residualized polygenic scores $(26,28)$. Population stratification is the non-random patterning of allele frequencies across ancestry groups. To quantify any such patterning, we estimated principal components from the genomewide SNP data for non-Hispanic white HRS respondents according to the method described by Price and colleagues (29) using the PLINK command pca (30). We then residualized the wellbeing polygenic score for the first 10 principal components, i.e. we regressed HRS respondents' polygenic scores on the 10 principal-component scores and computed residual values from the predictions. Residualized polygenic scores were standardized to $\mathrm{M}=0, \mathrm{SD}=1$ for analysis.

Death of Spouse. Death of one's spouse is a well-documented risk factor for developing depressive symptoms (31). During follow-up, $n=8,070(85 \%)$ of the sample were married and $\mathrm{n}=1,829$ (20\% of the sample, $24 \%$ of those married) experienced the death of their spouse. These survivors (74\% female, mean age 73 years) completed follow-up surveys on average 12 months after their spouse's death (IQR=6-18 months, Supplemental Figure 1).

Depressive Symptoms. Depressive symptoms were measured with the 8-item Centre for Epidemiological Studies Depression (CESD) scale at each HRS follow-up. The CESD is a valid psychometric instrument for assessment of depression in older adults $(32,33)$. We analyzed the continuous CESD score. There was substantial within-person variability in depressive symptoms across follow-up (ICC=0.48, $\mathrm{M}=1.2, \mathrm{SD}=1.8)$.

\section{Analysis}


Genetic heterogeneity in depressive symptoms following the death of a spouse

We conducted descriptive analysis of HRS respondents' depressive symptoms before and after the death of their spouses using non-parametric local regression. Local regression fits a smoothed curve to data that include nonlinear patterns by analyzing "localized" subsets of the data to build up a function iteratively (34). We used local regression to fit symptom data to timefrom-death. The resulting model describes symptom levels leading up to and following spousal death. If the death of one's spouse produces a change in depressive symptoms, this change will appear as a discontinuity in the local regression line estimated by the model, e.g. depressive symptom levels may "jump" from their trajectory before the death to a higher level after the death.

We conducted hypothesis tests using parametric nonlinear regression (35). Nonlinear regression estimates a function describing non-linear patterns in a set of data, and then models those nonlinearities as a function of other variables. Analysis modeled depressive symptoms at the first assessment following death of a spouse as a non-linear function of time since the death. We tested the hypothesis that wellbeing polygenic score would buffer against increase in depressive symptoms following death of a spouse using the coefficient for the association between polygenic score and that non-linear function.

Specifically, we estimated the model

$$
\mathrm{CESD}_{i}=\alpha+\beta_{1} \mathrm{PGS}_{i}+\left(\beta_{2}+\beta_{3} \mathrm{PGS}_{i}\right) \exp \left[t_{i} /\left(\lambda+\gamma \mathrm{PGS}_{i}\right)\right]+\text { controls }+\varepsilon_{i \cdot}(\text { Eqn } 1)
$$

$\alpha$ is the model intercept. $\mathcal{E}_{i}$ is a normally distributed random error term. $\beta_{l}$ estimates the main effect of the polygenic score on depressive symptoms. $\left(\beta_{2}+\beta_{3} \mathrm{PGS}_{i}\right) \exp \left[\mathrm{t}_{\mathrm{i}} /\left(\lambda+\gamma \mathrm{PGS}_{i}\right)\right]$ estimates the dynamics of depression in the wake of spousal death. $t_{i}$ is time since the death, 
Genetic heterogeneity in depressive symptoms following the death of a spouse

which is $>0$ for all observations. $\lambda+\gamma \mathrm{PGS}_{\mathrm{i}}$ is the "decay function" for the shock caused by the death. The decay function describes how the increase in depressive symptoms following death attenuates over time. The decay function has two components. $\lambda$ is the population average decay function. $\gamma$ describes how the decay function varies according to polygenic score. $\beta_{2}$ estimates the main effect of spousal death. $\beta_{3}$ tests the hypothesis that higher wellbeing polygenic score buffers against increases in depressive symptoms following spousal death. If $\beta_{3}<0$, we reject the null hypothesis that polygenic score is unrelated to depressive symptoms following spousal death. Models included controls for birth year, age, and sex. Graphical representation of model components is shown in Supplemental Figure 2.

Statistical analysis was conducted using the R software (36). We tested main-effect associations between HRS respondents' polygenic scores and their depressive symptoms using random-effects models implemented with the R package lme4 (37). We fit local regression models to repeated measures depressive symptom data using the loess function and the nonlinear regression models using the nls function.

\section{RESULTS}

\section{Higher wellbeing polygenic score was associated with reduced depressive symptoms.}

HRS respondents with higher polygenic scores experienced fewer depressive symptoms during follow-up $(b=-0.11, C I=-0.14,-0.08$, Figure 1). This effect-size is consistent with previous analysis of the wellbeing polygenic score and depression (16). The genetic association with depressive symptoms was similar for men and women $(\mathrm{p}=0.37$ for test of interaction between polygenic score and sex). 
Genetic heterogeneity in depressive symptoms following the death of a spouse

\section{Wellbeing polygenic score was not associated with likelihood of experiencing death}

of a spouse. Genetic liability to depression may influence exposure to stressful life events (38). Therefore, before testing the genetic buffering hypothesis, we evaluated possible geneenvironment correlation between subjective wellbeing polygenic score and likelihood of experiencing spousal death. We found no evidence of such gene-environment correlation; HRS respondents' polygenic scores were not related to their likelihood of experiencing the death of their spouse $(\mathrm{OR}=0.96, \mathrm{CI}=0.92-1.01, \mathrm{p}=0.145)$.

\section{Death of a spouse was associated with an immediate increase in depressive} symptoms, followed by a gradual return toward baseline over the following years. HRS respondents who experienced death of their spouse experienced a discontinuity in depressive symptoms around the time of the death. In local regression analysis, respondents' depressive symptoms trended slightly upwards in the months immediately preceding the death, rose sharply at the time of death, and gradually returned toward baseline over the following two years (Figure 2). This pattern of is consistent with previous reports on the course of depressive symptoms around spousal death (39).

Nonlinear regression analysis estimated the increase in CESD associated with spousal death to be 1.9 points (95\% CI 1.5-2.3). This increase was smaller for respondents interviewed farther from the time of their spouse's death. Based on trajectories estimated from the model, the increase in depressive symptoms that occurred following the death of a spouse attenuated by 12 months after the death, with modest further attenuation through 24 months (Supplemental Table 1). 
Genetic heterogeneity in depressive symptoms following the death of a spouse

\section{Genetics of subjective wellbeing buffered against increase in depressive symptoms}

following death of a spouse. HRS respondents with higher polygenic scores experienced flatter trajectories of depressive symptoms around the time of their spouse's death as compared to peers who also lost their spouse and had lower polygenic scores. In local regression analysis, HRS respondents with higher wellbeing polygenic scores experienced fewer depressive symptoms in the months leading up to the death and a smaller increase in depressive symptoms following the death as compared to other survivors who had lower polygenic scores (Figure 3).

In nonlinear regression analysis, each standard-deviation increase in polygenic score buffered the increase in depressive symptoms following spousal death by 0.46 CESD points (95\% CI 0.06, $0.86, \mathrm{p}=0.02)$. In context, this effect estimate suggests a person with a polygenic score 1SD above the population mean should experience about half as much increase in depressive symptoms following death of their spouse as compared to a peer whose polygenic score was $1 \mathrm{SD}$ below the population mean. Respondents' polygenic scores were not related to the rate of attenuation in depressive symptoms following the death $(\gamma=0.41, p=0.72$,

\section{Supplemental Figure 3, Supplemental Table 1).}

Sensitivity Analyses. We conducted sensitivity analyses to evaluate consistency of findings. First, we repeated analysis in a subset of HRS respondents identified as being of European descent based on analysis of their genetic data $(\mathrm{N}=1,647)$. (Our original analysis included all respondents who self-identified as non-Hispanic white.) Findings were unchanged in the genetically-defined European-descent sample (Supplemental Table 2).

Second, we repeated analysis using polygenic scores derived from two published GWAS of depression, the Psychiatric Genomics Consortium's GWAS of major depressive disorder (40) 
Genetic heterogeneity in depressive symptoms following the death of a spouse

and Social Science Genetic Association Consortium GWAS of depressive symptoms (16). The subjective wellbeing score was negatively correlated with depression polygenic scores $(\mathrm{r}=-0.16$ with the major depression score and $\mathrm{r}=-0.32$ with the depressive symptoms score; the major depression score and depressive symptom scores were correlated with each other, $\mathrm{r}=0.28$ ). Results from analysis of depression polygenic scores paralleled results from analysis of the subjective wellbeing polygenic score, but were in the opposite direction, reflecting the negative correlation between depression polygenic scores and subjective wellbeing polygenic score. HRS respondents with higher depression polygenic scores had elevated baseline levels of depressive symptoms and experienced a larger increase in depressive symptoms following the death of a spouse as compared to respondents with lower depression polygenic scores (Supplementary

Table 3).

\section{DISCUSSION}

We tested if genetics discovered in GWAS of subjective wellbeing buffered against development of depressive symptoms following death of a spouse. We analyzed data on depressive symptoms in 9,453 individuals followed longitudinally as part of the US Health and Retirement Study (HRS). During follow-up, 1,829 of these individuals experienced the death of their spouse. HRS respondents with higher polygenic scores experienced fewer depressive symptoms during follow-up. For those whose spouse died during the follow-up period, having higher polygenic score buffered against increased depressive symptoms following the death. Having a low polygenic score for depression had similar buffering effects on depression risk to 
Genetic heterogeneity in depressive symptoms following the death of a spouse

having a high polygenic score for subjective wellbeing. Magnitudes of genetic effects were small.

This evidence of genetic buffering against depression following a stressful life event provides proof of concept for further investigation of the genetics of subjective wellbeing as a modifier of the life-stress to depression link. Studies of gene-environment interplay are controversial (41). Statistical and theoretical models are contested (42-45). The precise nature of any "interaction" between the stress of losing a spouse and wellbeing-related genetic background remains uncertain. What we find is that individuals who carry more wellbeing-associated alleles tend to develop fewer additional depressive symptoms following the death of their spouse. This "buffering" is independent of the overall lower level of depressive symptoms associated with wellbeing polygenic score in the absence of a spousal death. Buffering is also not affected by any "gene-environment correlation" between genetics of subjective wellbeing and exposure to death of a spouse (46); HRS respondents' subjective wellbeing polygenic scores were unrelated to their risk of losing a spouse during follow-up. Finally, we detected consistent evidence of buffering using two different statistical specifications, local regression and nonlinear regression, that take into account time elapsed between the stressful event and the measurement of depressive symptoms.

Findings have implications for relevance of wellbeing genetics in clinical approaches to bereavement and for research into gene-environment interplay. Clinically, genetic testing based on current knowledge is unlikely to be useful in planning for or managing bereavement. Effect sizes in our study were too small to be useful for predicting outcomes of individual patients. It is possible that larger-scale GWAS will furnish more predictive polygenic scores with greater clinical relevance. However, even with very-large GWAS, predictive power of polygenic scores 
Genetic heterogeneity in depressive symptoms following the death of a spouse

for common, complex traits will be limited (47). Instead, our results may inform clinical research into candidate intervention targets to promote resilience in bereavement and in the context of other stressful experiences. Specifically, our findings raise questions about what behaviors or psychological processes mediate buffering effects. Do individuals with higher wellbeing polygenic scores process or cope with stress differently (48)? Do they cultivate different social support networks or interact with them in different ways? Studies to identify such mediators of genetic associations can inform interventions designed to promote resilience (49).

Regarding research on gene environment interplay, our results highlight two issues related to study design. First, our study suggests a model for integrating polygenic scores into studies of gene environment interplay: consider polygenic scores for characteristics theorized to modify effects of environmental exposures. Polygenic scores accumulate information from across the genome and take on normal distributions-properties that match theoretical models of human individual differences (50). But they are derived from GWAS that are naïve to environmental variation. Consequently, polygenic scores may quantify precisely those genetic influences that do not vary depending on environment as a byproduct of standard GWAS study design, although alternative designs are possible $(51,52)$. If correct, this explanation could account for null findings in previous studies of gene-environment interplay in depression that used polygenic scores derived from depression GWAS $(9,10)$. In contrast, we studied a polygenic score for a characteristic theorized to buffer environmental risks for depression. This "triangulation" approach may prove useful to future investigations. That said, our analysis of polygenic scores for depression yielded substantively identical results to our analysis of the polygenic score for subjective wellbeing. Thus, the observed gene-environment interplay is also consistent with a diathesis-stress model. Further investigation of overlap between genetic 
Genetic heterogeneity in depressive symptoms following the death of a spouse

underpinnings of subjective wellbeing and depression in the context of stressful life events is warranted.

A second design issue raised by our study is the temporal resolution of environmental exposure measures. Depression is an episodic condition. Even persistent cases experience temporary remissions. Increases in depressive symptoms following stressful life events may attenuate over time, as is characteristic in bereavement (53). Our study could account for this attenuation because precise dates were available for the timing of spousal death. We used statistical models designed for this type of data. Future studies may also wish to focus on stressful life events that can be located in specific temporal relation to the measurement of depressive symptoms (54).

This study has limitations. We studied depression in married older adults. Etiology of depression in older adults may differ in some ways from etiology in younger adults (55), although the relationship of stressful life events to depression does not seem to vary with age (56). Etiological features of depression, including subjective wellbeing may influence probability of marriage and of remaining married into later life (57). Replication of results in younger samples and with unmarried adults is needed. We studied depression following death of a spouse. Depression following death of a spouse may be different from depression related to other stressful life events. However, the distinction is unclear, as is reflected in the most recent revision of the American Psychiatric Association's Diagnostic and Statistical Manual of Mental Disorders, $5^{\text {th }}$ Edition (58). There is evidence that so-called bereavement-related depression shares etiology with depression related to other stressful life events (59). Tests of wellbeing polygenic score as a buffer against depressive symptoms following other types of stressful life events are needed. Our analysis was restricted to European-descent HRS participants. We 
Genetic heterogeneity in depressive symptoms following the death of a spouse

applied this restriction because of concerns about generalizability of GWAS results across racial/ethnic populations (24) and because the sample of spousal pairs of non-white HRS participants was too small for an independent analysis. Although relationships among stress, coping, and depression may be similar across ethnic groups (60), studies of the genetics of wellbeing and their potential role in buffering against depression following stressful life events in non-European populations are needed.

Research into interplay between genes and environments has a contentious history in psychiatry. With the advent of large-scale GWAS, new, more reliable genetic measures of liability to psychiatric disorders and other mental health characteristics are emerging. At the same time, genetic data are becoming available for large, population-based social surveys that have recorded environmental exposure information for participants. Together, these resources should enable a new generation of molecular genetic research into gene-environment interplay research in the etiology of psychiatric disorders.

Conflict of Interest: The authors report no conflict of interest.

Acknowledgements: This research uses data from the HRS, which is sponsored by the National Institute on Aging (Grants NIA U01AG009740, RC2AG036495, and RC4AG039029) and conducted by the University of Michigan. Further support was provided by the NIH/NICHDfunded University of Colorado Population Center (R24HD066613). DWB is supported by an Early Career Research Fellowship from the Jacobs Foundation and NIA grants P30AG034424 and P30AG028716. AO is supported by ERC Consolidator Grant (647648 EdGe). This research was facilitated by the Social Science Genetic Association Consortium (SSGAC). 
bioRxiv preprint doi: https://doi.org/10.1101/065847; this version posted September 21, 2016. The copyright holder for this preprint (which was not certified by peer review) is the author/funder, who has granted bioRxiv a license to display the preprint in perpetuity. It is made available under aCC-BY-NC-ND 4.0 International license.

Genetic heterogeneity in depressive symptoms following the death of a spouse

\section{References}

1. Kessler RC. The Effects of Stressful Life Events on Depression. Annu Rev Psychol. 1997;48(1):191-214.

2. Kendler KS, Karkowski LM, Prescott CA. Causal Relationship Between Stressful Life Events and the Onset of Major Depression. Am J Psychiatry. 1999 Jun 1;156(6):837-41.

3. Monroe SM, Simons AD. Diathesis-stress theories in the context of life stress research: Implications for the depressive disorders. Psychol Bull. 1991;110(3):406-25.

4. Silberg J, Rutter M, Neale M, Eaves L. Genetic moderation of environmental risk for depression and anxiety in adolescent girls. Br J Psychiatry. 2001 Aug 1;179(2):116-21.

5. Kendler KS, Kessler RC, Walters EE, MacLean C, Neale MC, Heath AC, et al. Stressful life events, genetic liability, and onset of an episode of major depression in women. Am J Psychiatry. 1995;

6. Caspi A, Hariri AR, Holmes A, Uher R, Moffitt TE. Genetic Sensitivity to the Environment: The Case of the Serotonin Transporter Gene and Its Implications for Studying Complex Diseases and Traits. Am J Psychiatry. 2010 May 1;167(5):509-27.

7. Belsky DW, Suppli NP, Israel S. Gene-environment interaction research in psychiatric epidemiology: a framework and implications for study design. Soc Psychiatry Psychiatr Epidemiol. 2014 Sep 13;49(10):1525-9.

8. Peyrot WJ, Milaneschi Y, Abdellaoui A, Sullivan PF, Hottenga JJ, Boomsma DI, et al. Effect of polygenic risk scores on depression in childhood trauma. Br J Psychiatry. 2014 Aug 1;205(2):113-9.

9. Mullins N, Power RA, Fisher HL, Hanscombe KB, Euesden J, Iniesta R, et al. Polygenic interactions with environmental adversity in the aetiology of major depressive disorder. Psychol Med. 2016 Mar;46(4):759-770.

10. Musliner KL, Seifuddin F, Judy JA, Pirooznia M, Goes FS, Zandi PP. Polygenic risk, stressful life events and depressive symptoms in older adults: a polygenic score analysis. Psychol Med. 2015 Jun;45(8):1709-1720.

11. Diener E. Subjective well-being. The science of happiness and a proposal for a national index. Am Psychol. 2000 Jan;55(1):34-43.

12. Keyes CL. The mental health continuum: From languishing to flourishing in life. J Health Soc Behav. 2002;207-222.

13. Weiss A, Bates TC, Luciano M. Happiness is a personal (ity) thing the genetics of personality and well-being in a representative sample. Psychol Sci. 2008;19(3):205-210. 
Genetic heterogeneity in depressive symptoms following the death of a spouse

14. Rietveld CA, Cesarini D, Benjamin DJ, Koellinger PD, Neve J-ED, Tiemeier H, et al. Molecular genetics and subjective well-being. Proc Natl Acad Sci. 2013 Jun 11;110(24):9692-7.

15. Bartels M, Cacioppo JT, Beijsterveldt TCEM van, Boomsma DI. Exploring the Association Between Well-Being and Psychopathology in Adolescents. Behav Genet. 2013 Mar 8;43(3):177-90.

16. Okbay A, Baselmans BML, De Neve J-E, Turley P, Nivard MG, Fontana MA, et al. Genetic variants associated with subjective well-being, depressive symptoms, and neuroticism identified through genome-wide analyses. Nat Genet [Internet]. 2016 Apr 18 [cited 2016 May 3]; advance online publication. Available from: http://www.nature.com/ng/journal/vaop/ncurrent/full/ng.3552.html

17. Park N. The Role of Subjective Well-Being in Positive Youth Development. Ann Am Acad Pol Soc Sci. 2004 Jan 1;591(1):25-39.

18. Grant F, Guille C, Sen S. Well-being and the risk of depression under stress. PLoS One. 2013;8(7):e67395.

19. Juster FT, Suzman R. An Overview of the Health and Retirement Study. J Hum Resour. 1995;30:S7-56.

20. Sonnega A, Faul JD, Ofstedal MB, Langa KM, Phillips JW, Weir DR. Cohort Profile: the Health and Retirement Study (HRS). Int J Epidemiol. 2014 Apr 1;43(2):576-85.

21. Chien S, Campbell N, Hayden O, Hurd M, Main R, Mallett J, et al. RAND HRS Data Documentation, Version N. 2014;

22. Domingue BW, Belsky DW, Conley D, Harris KM, Boardman JD. Polygenic Influence on Educational Attainment. AERA Open. 2015;1(3):2332858415599972.

23. Belsky DW, Moffitt TE, Sugden K, Williams B, Houts R, McCarthy J, et al. Development and evaluation of a genetic risk score for obesity. Biodemography Soc Biol. 2013;59(1):85100 .

24. Carlson CS, Matise TC, North KE, Haiman CA, Fesinmeyer MD, Buyske S, et al. Generalization and dilution of association results from European GWAS in populations of non-European ancestry: the PAGE study. PLoS Biol. 2013;11(9):e1001661.

25. Dudbridge F. Power and predictive accuracy of polygenic risk scores. PLoS Genet. 2013;9(3):e1003348.

26. Conley D, Laidley T, Belsky D, Fletcher J, Boardman J, Domingue B. Assortative mating and differential fertility by phenotype and genotype across the 20th century. Proc Natl Acad Sci. 2016; 
Genetic heterogeneity in depressive symptoms following the death of a spouse

27. Domingue BW, Conley D, Fletcher J, Boardman JD. Cohort Effects in the Genetic Influence on Smoking. Behav Genet. 2015;1-12.

28. Selzam S, Krapohl E, von Stumm S, O’Reilly PF, Rimfeld K, Kovas Y, et al. Predicting educational achievement from DNA. Mol Psychiatry [Internet]. 2016 Jul 19; Available from: http://dx.doi.org/10.1038/mp.2016.107

29. Price AL, Patterson NJ, Plenge RM, Weinblatt ME, Shadick NA, Reich D. Principal components analysis corrects for stratification in genome-wide association studies. Nat Genet. 2006;38(8):904-909.

30. Chang CC, Chow CC, Tellier LC, Vattikuti S, Purcell SM, Lee JJ. Second-generation PLINK: rising to the challenge of larger and richer datasets. GigaScience [Internet]. 2015 Feb 25 [cited 2015 Oct 19];4. Available from: http://www.ncbi.nlm.nih.gov/pmc/articles/PMC4342193/

31. Zisook S, Shuchter SR. Depression through the first year after the death of a spouse. Am J Psychiatry. 1991;148(10):1346-1352.

32. Irwin M, Artin KH, Oxman MN. Screening for depression in the older adult: criterion validity of the 10-item Center for Epidemiological Studies Depression Scale (CES-D). Arch Intern Med. 1999 Aug 9;159(15):1701-4.

33. Lyness JM, Noel TK, Cox C, King DA, Conwell Y, Caine ED. Screening for depression in elderly primary care patients. A comparison of the Center for Epidemiologic StudiesDepression Scale and the Geriatric Depression Scale. Arch Intern Med. 1997 Feb 24;157(4):449-54.

34. Cleveland WS. Robust locally weighted regression and smoothing scatterplots. J Am Stat Assoc. 1979;74(368):829-36.

35. Sánchez BN, Wu M, Raghunathan TE, Diez-Roux AV. Modeling the Salivary Cortisol Profile in Population Research The Multi-Ethnic Study of Atherosclerosis. Am J Epidemiol. 2012;kws182.

36. R Core Team. R: A Language and Environment for Statistical Computing [Internet]. Vienna, Austria: R Foundation for Statistical Computing; 2016. Available from: https://www.R-project.org/

37. Bates D, Maechler M, Bolker B. lme4: Linear mixed-effects models using S4 classes [Internet]. 2013. Available from: http://CRAN.R-project.org/package=lme4

38. Kendler KS, Karkowski-Shuman L. Stressful life events and genetic liability to major depression: genetic control of exposure to the environment? Psychol Med. 1997 May;27(3):539-547. 
Genetic heterogeneity in depressive symptoms following the death of a spouse

39. Turvey CL, Carney C, Arndt S, Wallace RB, Herzog R. Conjugal Loss and Syndromal Depression in a Sample of Elders Aged 70 Years or Older. Am J Psychiatry. 1999 Oct $1 ; 156(10): 1596-601$.

40. Ripke S, Wray NR, Lewis CM, Hamilton SP, Weissman MM, Breen G, et al. A megaanalysis of genome-wide association studies for major depressive disorder. Mol Psychiatry. 2013;18(4):497-511.

41. Uher R. Gene-environment interactions in severe mental illness. Front Psychiatry. 2014;5:48.

42. VanderWeele TJ. Sufficient cause interactions and statistical interactions. Epidemiology. 2009;20(1):6-13.

43. Keller MC. Gene $\times$ Environment Interaction Studies Have Not Properly Controlled for Potential Confounders: The Problem and the (Simple) Solution. Biol Psychiatry. 2014 Jan $1 ; 75(1): 18-24$.

44. Moffitt TE, Caspi A, Rutter M. Measured Gene-Environment Interactions in Psychopathology Concepts, Research Strategies, and Implications for Research, Intervention, and Public Understanding of Genetics. Perspect Psychol Sci. 2006 Mar $1 ; 1(1): 5-27$.

45. Belsky J, Bakermans-Kranenburg MJ, IJzendoorn MH van. For Better and For Worse Differential Susceptibility to Environmental Influences. Curr Dir Psychol Sci. 2007 Dec $1 ; 16(6): 300-4$.

46. Jaffee SR, Price TS. Gene-environment correlations: a review of the evidence and implications for prevention of mental illness. Mol Psychiatry. 2007;12(5):432-442.

47. Wray NR, Yang J, Hayes BJ, Price AL, Goddard ME, Visscher PM. Pitfalls of predicting complex traits from SNPs. Nat Rev Genet. 2013 Jul;14(7):507-15.

48. Gotlib IH, Joormann J, Foland-Ross LC. Understanding familial risk for depression a 25year perspective. Perspect Psychol Sci. 2014;9(1):94-108.

49. Belsky DW, Moffitt TE, Caspi A. Genetics in Population Health Science: Strategies and Opportunities. Am J Public Health. 2013 Aug 8;103(S1):S73-83.

50. Plomin R, Haworth CM, Davis OS. Common disorders are quantitative traits. Nat Rev Genet. 2009;10(12):872-878.

51. Boardman JD, Domingue BW, Blalock CL, Haberstick BC, Harris KM, McQueen MB. Is the gene-environment interaction paradigm relevant to genome-wide studies? The case of education and body mass index. Demography. 2014;51(1):119-139. 
Genetic heterogeneity in depressive symptoms following the death of a spouse

52. Yang J, Loos RJF, Powell JE, Medland SE, Speliotes EK, Chasman DI, et al. FTO genotype is associated with phenotypic variability of body mass index. Nature. 2012 Oct 11;490(7419):267-72.

53. Zisook S, Paulus M, Shuchter SR, Judd LL. The many faces of depression following spousal bereavement. J Affect Disord. 1997 Aug 1;45(1-2):85-95.

54. Sen S, Kranzler HR, Krystal JH, Speller H, Chan G, Gelernter J, et al. A prospective cohort study investigating factors associated with depression during medical internship. Arch Gen Psychiatry. 2010;67(6):557-565.

55. Blazer DG. Depression in late life: review and commentary. J Gerontol A Biol Sci Med Sci. 2003;58(3):M249-M265.

56. Kessing LV, Agerbo E, Mortensen PB. Does the impact of major stressful life events on the risk of developing depression change throughout life? Psychol Med. 2003;33(7):11771184.

57. Mastekaasa A. Marriage and psychological well-being: Some evidence on selection into marriage. J Marriage Fam. 1992;901-911.

58. American Psychiatric Association, others. Diagnostic and statistical manual of mental disorders (DSM-5®). American Psychiatric Pub; 2013.

59. Kendler KS, Myers J, Zisook S. Does bereavement-related major depression differ from major depression associated with other stressful life events? Am J Psychiatry. 2008;165(11):1449-1455.

60. Keyes KM, Barnes DM, Bates LM. Stress, coping, and depression: testing a new hypothesis in a prospectively studied general population sample of US-born Whites and Blacks. Soc Sci Med. 2011;72(5):650-659. 
Genetic heterogeneity in depressive symptoms following the death of a spouse

\section{Figure Legends}

Figure 1. HRS respondents with higher polygenic scores reported fewer depressive symptoms during follow-up. The figure shows average polygenic scores (denominated in standard deviation units) for groups of HRS respondents defined by their histories of depressive symptoms. Depressive symptom history was measured by averaging respondents' CESD scores across repeated assessments and rounded to the nearest integer value. The resulting groups included 3,757 respondents with an average score of $0,2,819$ with a score of $1,1,470$ with a score of 2, 702 with a score of 3 , and 705 with a score of 4 or higher.

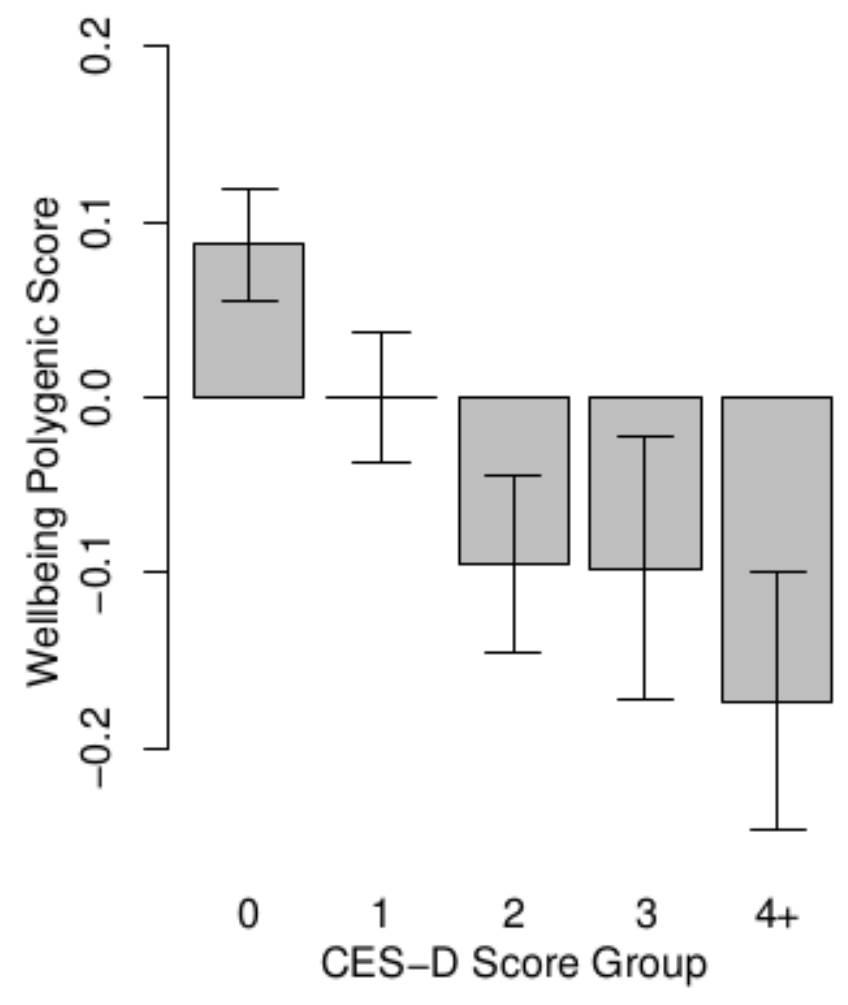


Genetic heterogeneity in depressive symptoms following the death of a spouse

Figure 2. Trajectories of depressive symptoms in the months surrounding the death of a spouse. The figure shows local regression plots of depressive symptoms (CESD scores) by month of measurement relative to the death of a spouse. The plot reflects data from HRS respondents who experience spousal death $(\mathrm{N}=1,829 ; 15,853$ total observations). Plotted points show mean $\mathrm{x}$ - and $\mathrm{y}$ - coordinates for bins of about 40 individuals.

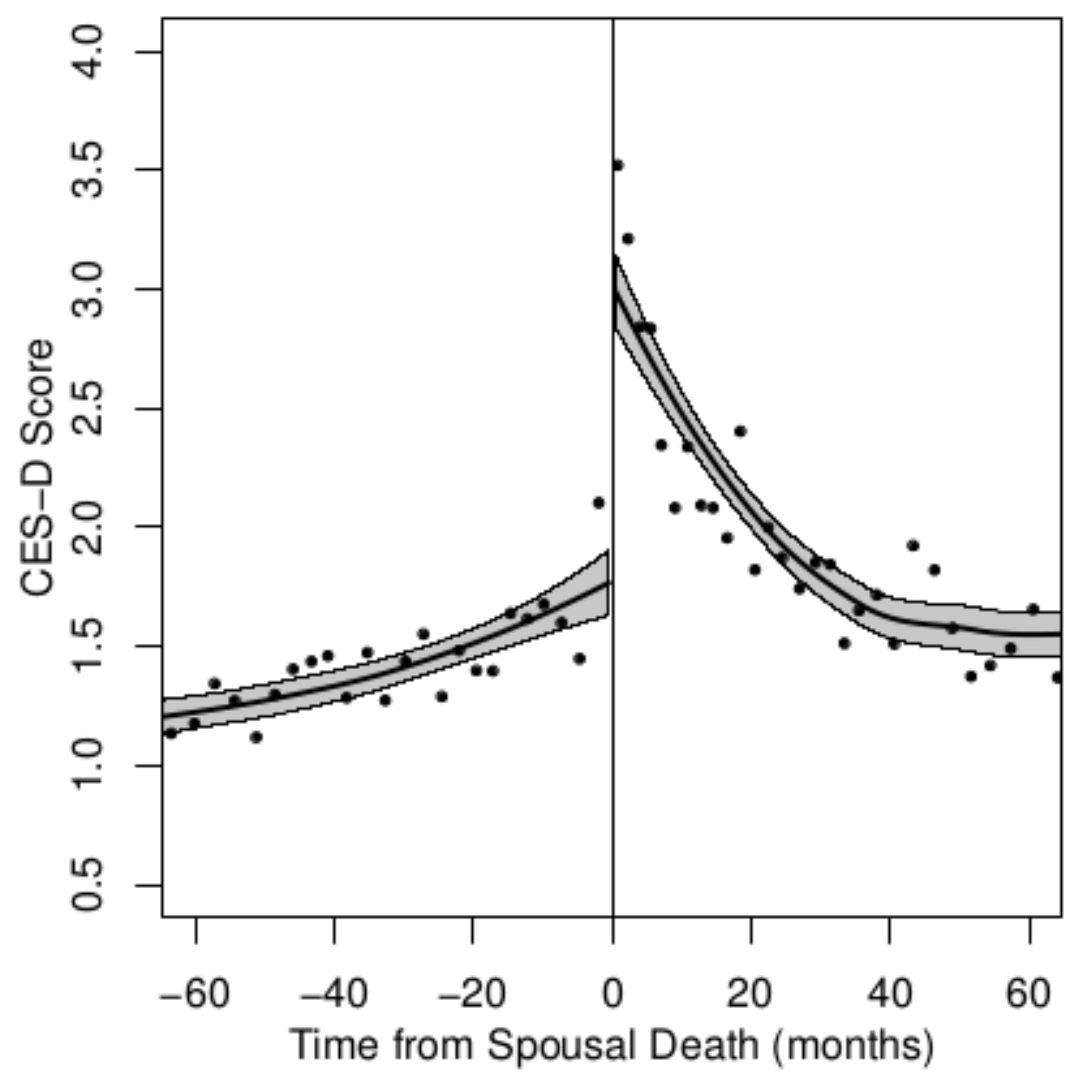


bioRxiv preprint doi: https://doi.org/10.1101/065847; this version posted September 21,2016 . The copyright holder for this preprint (which was not certified by peer review) is the author/funder, who has granted bioRxiv a license to display the preprint in perpetuity. It is made available under aCC-BY-NC-ND 4.0 International license.

Genetic heterogeneity in depressive symptoms following the death of a spouse

Figure 3. Trajectories of depressive symptoms for HRS respondents with low polygenic scores (red line) and high polygenic scores (blue line). The figure shows local regression plots of depressive symptoms (CESD Scores) by month of measurement relative to the death of a spouse. Trajectories for respondents with low polygenic scores (1 or more SDs below the mean, $\mathrm{n}=2,493$ ) are graphed in red. Trajectories for HRS respondents with high polygenic scores ( 1 or more SDs above the mean, $n=2,181$ ) are graphed in blue.

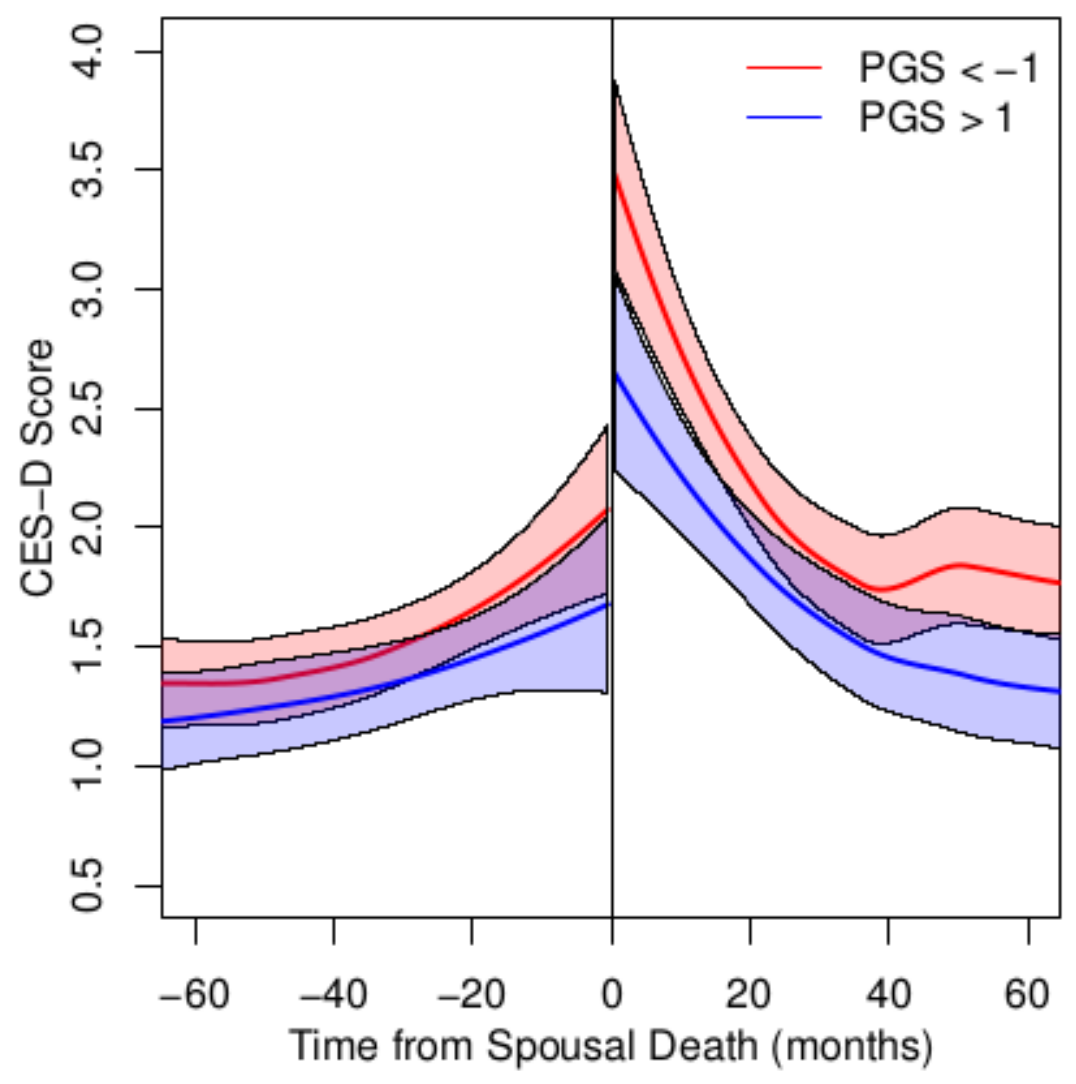

